\title{
Simulation Analysis for Multicast Context Delivery Network Mobility Management
}

\author{
Azana Hafizah Mohd Aman, Aisha-Hassan A. Hashim*, Huda Adibah Mohd Ramli \\ Kulliyyah of Engineering, International Islamic University Malaysia \\ Jalan Gombak 53100, Kuala Lumpur, Malaysia \\ e-mail: aisha@iium.edu.my*, azana05@yahoo.com
}

\begin{abstract}
The objective of this paper is to presents analyses for multicast network mobility management using NS3. It is mainly to verify the proposed network architecture and its activities. NS3 is a network simulator that implements virtually network prototype that is close to real implementation. Network mobility management has become a popular topic in networking research due to its ability to mitigate mobile IPv6 problems. However the standard network mobility management only introduced to support unicast traffic. Hence this paper integrates context transfer and multicast fast reroute, and implements this integration to the standard network mobility management. This implementation enables multicast to network mobility management with high network performance support. The analyses focus on the throughput performance. The analyses of this simulator are hereby presented.
\end{abstract}

Keywords: Multicast, Network Mobility, Mobile Multicast, Network Mobility Management

\section{Introduction}

In 2019, IP traffic will surpass two zettabyte threshold with mobile data traffic that grows three times quicker than fixed IP traffic $[1,2,3]$. Multicast mobility IP has been an interest in recent years but there is as yet no standard solution $[4,5]$.

The method of changing network layer while moving for the standard Mobility in IPv6 (MIPv6) [6] suffers from slow handovers due to multicast routing and inefficient overhead as well as delay due to triangular forwarding $[4,5]$. As a resolution to this problem, this paper offered an improvement to support high data delivery performance in network mobility management. The improved method is an implement of integration of two concepts into Proxy Mobile Ipv6 (PMIPv6) [7, 8]. In the offered method the mobile multicast traffic is processed and delivered using the integration of Context Transfer [9, 10,11] and Multicast Fast Reroute [12]. The novel improved method is named as CTMFR. This integration improves handover performance with shortened process of delivering the required multicast input. This paper evaluates the offered improvement using Network Simulator known as NS3. The offered improvement is benchmarked with the standard method [13] of PMIPv6 (proposed by IETF).

This paper is organized as follows. Section 2 describes recent network mobility management development, then section 3 the proposed improvement method. This is followed by the simulation results and analysis in section 4 . As a final point, the conclusion of the paper is presented in Section 5.

\section{Recent Network Mobility Management Development}

Generally a network simulator is an application that predicts the events of a network $[14,15]$. Network devices are depicted with nodes, links, devices and etc. While simulation is a testing of the depicted network entities on a network simulator [14, 15]. Simulation of network is difficult, as it is not simple to develop network event, such as handover, multicast or mobility. A network emulator allows users to host real devices and applications into a virtual test network $[14,15]$. A network simulator must be able to model and configure network entities, topologies and able to provide network results in values and graphs.

In [16], the performance of PMIPv6 protocol is studied using Network Simulator (NS) version 2.29. It extends existing MIPv6 to enable PMIPv6 environment. Total number of 7 nodes including 1 mobile node was configured. User Datagram Protocol (UDP) and Transmission Control Protocol (TCP) were set as traffic. Performance metrics measured are throughput, 
average handover delay and average packet delivery ratio. Data collected are number of packets received, number of packets sent and number of bytes receive. Even though it verified that the proposed architecture satisfied the flow mobility requirements of PMIPv6, there is no multicast traffic included.

Multi-Protocol Label Switching (MPLS) using OMNET++ network simulation for PMIPv6 is done in work [17]. Some modification was done using XMIPv6 as a base to support the MPLS/PMIPv6 protocol. Performance metrics considered are end-to-end delay, handover overhead and delay. Variety number of hop between MAG and LMA were set for the simulation. Scenario manager, configurator and channel control modules of OMNET++ are used to support the network model. It verified that the proposed architecture satisfied the flow mobility standards of PMIPv6. Work in [19] improved flow mobility support using the NS3 network simulator. By using these NS3 features, it implemented the multi-interfaced MNs and the flow mobility procedure in the proposed architecture. It verified that the proposed architecture fulfilled the flow mobility standards.

As for [18] and [20] it only covers the security part of network mobility management. Hence [18] implemented authorization, authentication and accounting (AAA) server in PMIPv6 topology. The AAA server and PMIPv6 implementation is via NS-2.29. Performance metric considered are handover delay and handover. Results are calculated based on varying number of LMAs. It verified that the proposed architecture and scheme satisfy the flow mobility requirements of PMIPv6. While work in [20], suggested a secure handover mechanism for PMIPv6 environment. It executed group key ticket for fast re-authentication using NS2. Performance metrics measured are packet loss, handover latency and signaling cost. It proved that the proposed architecture pleased the flow mobility standards of PMIPv6.

Using NS3 work in [21] described PMIPv6 environment. It applied MIPv6 module in NS3 as the basic module. Configured the User Datagram Protocol (UDP) and Constant Bit Rate Protocol (CBR) as the traffics. Performance metric measured are packet drop and handover latency. The values are collected in a module called PCAP. Another [22] considered the performance of PMIPv6 protocol using QUALNET. In order to apply PMIPv6 module, it implemented two-phase traffic control by extending the queuing discipline of the QUALNET. Performance metrics considered are packet buffering and packet loss. Even though work in [21] and [22] satisfied the flow mobility requirements of PMIPv6, there is no multicast traffic included.

\section{Proposed Multicast Network Mobility Management}

Improving PMIPv6 with multicast support ability is an ongoing concept to study. This is because, the PMIPv6 basically concerns on the unicast mobility support and little considers the multicast mobility. This delivers the objective of this paper which is to integrate context transfer and multicast fast reroute, and implements this integration to the standard network mobility management. This implementation enables multicast to network mobility management with high network performance support. Besides enabling multicast, this method address the handover performance issues related to PMIPv6 multicast environment.

Using this method, the context is transferred in advanced. This reduces unnecessary transmission after the handover. Mobile Node $(\mathrm{MN})$ is able to receive multicast data immediately after the handover.

From the above mentioned operation, it can be seen that by applying the integration, the time needed to re-establish the service can be reduced. This is due to the fact that, the Multicast context transfer block is transferred between the two MAGs (M-MAGs) before the handover is completed. Hence, all the information needed for the MN to join the multicast group is already transferred. The $\mathrm{MN}$ can then join the multicast group as soon as the MN moves to the nMAG. Also the signaling cost is reduced since the communication is localized between the two MAGs. The mobile node doesn't involve in sending the group membership message to the nMAG or LMA. The nMAG receives the information needed in the multicast context transfer block that is sent by pMAG.

Figure 1 shows the network topology detail for the simulation, while figure 4 shows the NS3 network topology setup according to Figure 2 with total number of 18 nodes including 1 MN. 


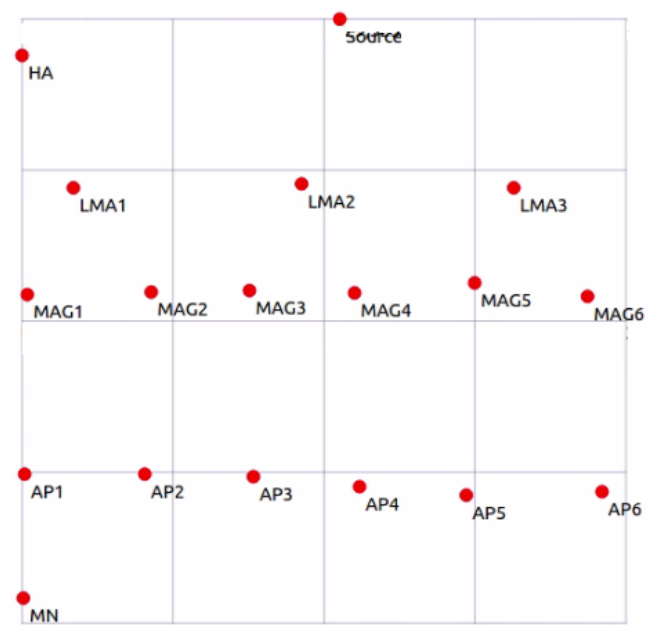

Figure 1. Network topology for the simulation

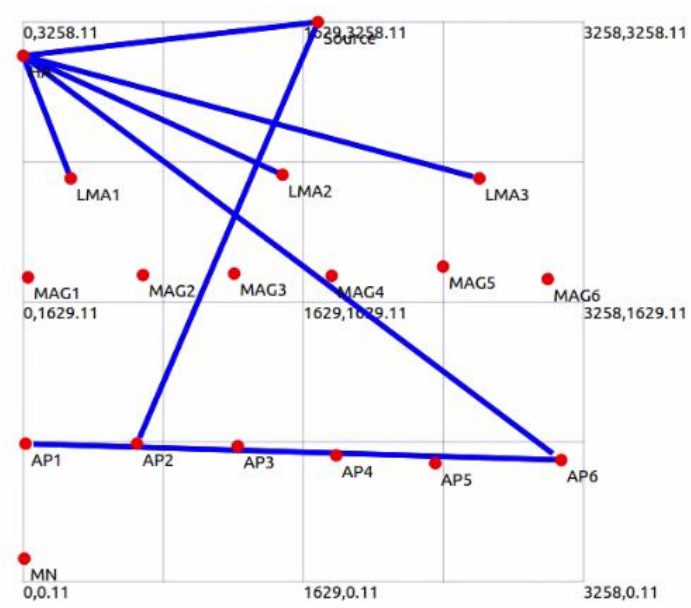

Figure 2. Simulation Topology Setup

\section{Simulation Results and Analysis}

Node mobility certainly has a significant impact upon network performance. When a mobile node with an active flow moves from one network to another network, the path for dataflow also changes. The throughput values are extracted from the trace-file produced during the simulation process using a trace-file analyzer.

To achieve seamless mobility with high performance, the throughput value should be kept as high as possible. Low throughput may affect real-time interactive applications including voice and video calls, multimedia applications, online gaming, etc [23]. The amount of acceptable throughput degradation depends on specific application since their acceptable rate differs from each other. For some applications, throughput below a certain limits may cause service disconnection, leads to poor handover performance. The relationship between throughput and network performance is in parallel to each other where the higher the throughput is, the higher the performance, thus requires more sophisticated and promising means to overcome the problem.

Figure 4 and Figure 5, show the throughput and UDP datagram ID for the improvise method and for the standard method. This proved that by using CTMFR, the throughput is better. 


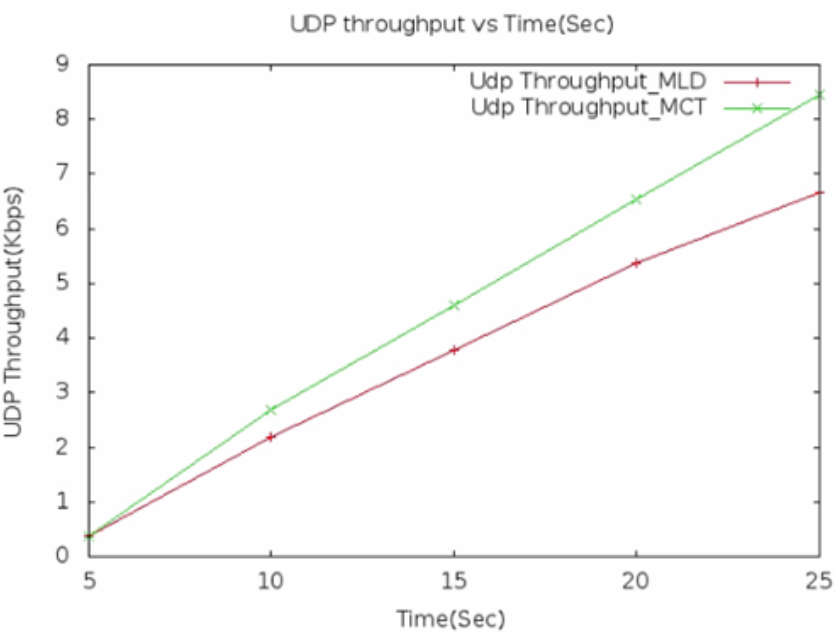

Figure 4. UDP Throughput versus Time

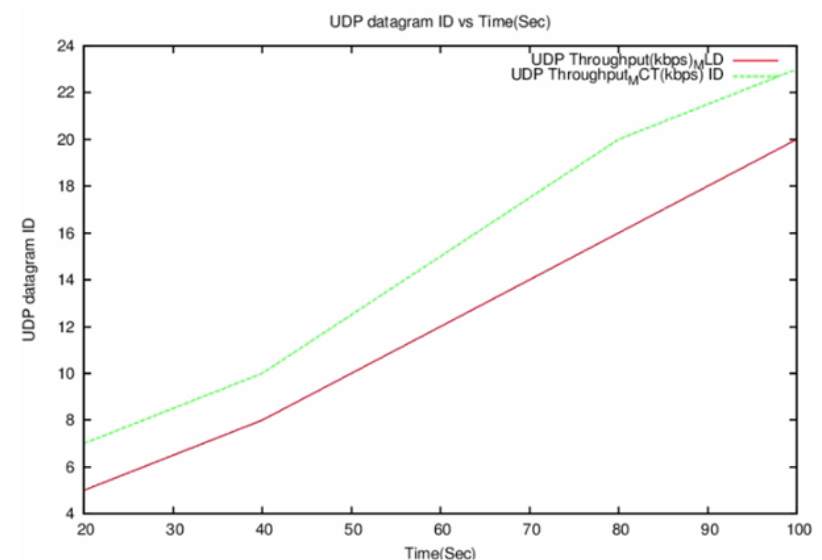

Figure 5 UDP Datagram ID versus Time

The effectiveness of the applied method has been shown in the Figure 4 and Figure 5 . It can be seen that there is huge gap between the improvised method and the standard method. By using CTMFR, the throughput performance is almost $50 \%$ better than the standard method.

The fast reroute and context transfer simplifies the path, and this leads to better throughput performance. This enhancement enables two-path context transfer design in PMIPv6 multicast. Multicast traffics, especially for the real-time video applications which are densely watched channels, typically flow along both the path in the network. Since both MAGs are already in the same multicast group. Hence, there will be smooth flow with no connection break, and thus traffic can continuously running without any interruption.

\section{Conclusion}

The improvised method is evaluated using simulation approach. After describing the simulation setup, the performance results of the selected metrics are presented and validated. Compared to PMIPv6 standard method, the improvised method offers highly considerable better throughput performance. From the simulation results, this proved that by applying the predictive CT and MFR, the enhanced method is more reliable than PMIPv6 and costs less performance degradation compared to the PMIPv6 method. 


\section{References}

[1] Cisco Visual Networking Index: Global Mobile Data Traffic Forecast Update 2014 - 2019. Cisco White Paper. 2015.

[2] Cisco Visual Networking Index: Forecast and Methodology 2014 - 2019. Cisco White Paper. 2015.

[3] Cisco Visual Networking Index: The Zettabyte Era Trends and Analysis. Cisco White Paper. 2015.

[4] TC Schmidt, M Waehlisch, G Fairhurst. Multicast mobility in MIPv6: problem statement \& brief survey. RFC 5757. 2010.

[5] CJ Bernardos, M Calderon, I Soto. PMIPv6 and network mobility problem statement. Internet-Draft. 2012.

[6] Johnson D, Perkins C, J Arkko. Mobility support in IPv6. RFC 3775. 2004.

[7] Gundavelli S, Ed Leung, K Devarapalli, V Chowdhury K, B Patil. Proxy Mobile IPv6(PMIPv6). RFC 5213. 2008

[8] Daehyeok Kim, Wan-Seon Lim, Young-Joo Suh. Multicast extension to Proxy Mobile IPv6 for mobile multicast services. Journal of Computing Science and Engineering. 2011: 316-323.

[9] D von Hugo, H Asaeda. Context Transfer Protocol extension for multicast. Internet-Draft. 2013.

[10] J Loughney, M Nakhjiri, C Perkins, R Koodli. Context Transfer Protocol. RFC 4067. 2005.

[11] Tien-Thinh Nguyen, Christian Bonnet. Performance optimization of multicast content delivery in a mobile environment based on PMIPv6. Wireless Communications and Networking Conference (WCNC) IEEE, 7-10 April 2013: 1249-1254.

[12] A Karan, C Filsfils, IJ Wijnands, B Decraene. Multicast Only Fasr Reroute (MoFRR). RFC 7431. 2015.

[13] T Schmidt, M Waehlisch, S Krishnan. Base deployment for multicast listener support in Proxy Mobile IPv6 (PMIPv6) domains. RFC 6224. 2011.

[14] Saba Siraj, Ajay Kumar Gupta, Rinku-Badgujar. Network Simulation Tools Survey, International J. of Advanced Research in Computer and Communication Engineering. 2012; 1(4).

[15] Jianli Pan. A Survey of Network Simulation Tools: Current Status and Future Developments.

[16] Farouk Abdul Jalin, Raed Alsaqour. A Simulation Study of Proxy Mobile IPV6 (PMIPV6) Protocol, Journal of Engineering and Applied Sciences. 2016; 11(7).

[17] Hatsadin Payappanon, Thossaporn Kamolphiwong, Kevin Robert Elz. Simulation and Evaluation of MPLS based PMIPv6 Network. International J. of Advances in Computer Science and Technology. 2013; 2(8): 07-11.

[18] Ashwini Prakash Ghatol, NM Tarbani. Optimized Handover Mechanism for Proxy Mobile IPv6. International Journal of Advanced Research in Computer Science and Software Engineering IJARCSE. 2015; 5(6).

[19] Hyon-Young Choi, Sung-Gi Min, Youn-Hee Han and Rajeev Koodli, Design and Simulation of a Flow Mobility Scheme Based on Proxy Mobile IPv6. J Inf Process Syst. 2012; 8(4).

[20] K Mayuri, KS Ranjith. A Novel Secure Handover Mechanism In Pmipv6 Networks. International J. of Information Technology Convergence and Services. 2014; 4(4).

[21] Hyon-Young Choi, Sung-Gi Min, Youn-Hee Han, Jungsoo Park, Hyoungjun Kim. Implementation and Evaluation of Proxy Mobile IPv6 in NS-3 Network Simulator. IEEE Xplore. 2011.

[22] Takeshi Usui. Designing Improved Traffic Control in Network-based Seamless Mobility Management for Wireless LAN. The Third International Conference on Advances in Future Internet. 2011.

[23] Muhamad Hanif Jofri, Mohd Farhan Md Fudzee, Mohd Norasri Ismail, Shahreen Kasim, Jemal Abawajy. Quality of Experience (QOE) Aware Video Attributes Determination for Mobile Streaming Using Hybrid Profiling. Indonesian Journal of Electrical Engineering and Computer Science. 2017; 8(3): 597-608. 